\title{
Identifying the Most Appropriate Intervention Targets Using Prediction Model Based on a Machine-Learning Method: A Retrospective Analysis of a Health Promotion Program for Improving Participation in General Health Check-Up
}

\author{
Akihiro Shimoda ${ }^{1 *}$, Yoshiyuki Saito ${ }^{2,3}$, Chieko Ooe ${ }^{4}$, Daisuke Ichikawa ${ }^{5}$, Ataru Igarashi ${ }^{2}$, Takeo Nakayama ${ }^{3}$, \\ Toki Saito ${ }^{1}$ and Hiroshi Oyama ${ }^{1,5}$ \\ ${ }^{1}$ Department of Clinical Information Engineering, Division of Social Medicine, Graduate School of Medicine, \\ The University of Tokyo, Tokyo, Japan \\ ${ }^{2}$ Department of Health Economics \& Outcomes Research, Graduate School of Pharmaceutical Sciences, \\ The University of Tokyo, Tokyo, Japan \\ ${ }^{3}$ Department of Health Informatics, School of Public Health, Graduate School of Medicine, Kyoto University, \\ Kyoto, Japan \\ ${ }^{4}$ Planning and Administration Group, Fukuoka Branch of Japan Health Insurance Association, Fukuoka, Japan \\ ${ }^{5}$ Department of Clinical Information Engineering, School of Public Health, Graduate School of Medicine, \\ The University of Tokyo, Tokyo, Japan
}

\begin{abstract}
Objective: We aimed to identify the population in which encouraging participation in the general health check-up would be helpful using a prediction model based on a machine-learning method. A secondary analysis of data obtained from the health promotion program using a randomized controlled design, aimed at improving participation in the general health check-up, was performed.
\end{abstract}

Methods: The retrospective analysis was conducted using data from a health promotion program in the Fukuoka branch of Japan Health Insurance Association, Japan, between November 2015 and March 2016. Subjects were extracted from dependents (family members) of insured persons aged $40-74$ years who had participated in general health check-up at least once in the past five years (2010-2014). Subjects were divided into two groups; the intervention group received a printed reminder saying "you are due to general health check-up" through mail, while the control group received nothing. The participation rates of both groups for each participation probability group (participation probability was calculated by the prediction model) were assessed after 4 month follow-up.

Results: The numbers in the intervention group and in the control group were 1,911 and 3,294, respectively. Regarding the prediction model, the AUC value for test data was 0.668 (95\% $\mathrm{Cl}: 0.635-0.701)$. With regard to the effectiveness of the intervention for each probability group, there was a significant difference between the groups only for the moderate participation probability groups as follows: $30-39 \%(P=0.005), 40-49 \%(P=0.003), 50-59 \%$ $(P=0.004)$ and $60-69 \%(P=0.039)$.

Conclusion: The intervention with printed reminder was effective for improving participation of general health check-up among the group with moderate participation probability. The targeting using the results of prediction model was useful for identifying appropriate intervention targets.

Conclusions: More studies are needed to assess the cost and benefits of adopting a system like this and then the appropriate actions could be taken.

\section{Keywords}

Mass screening; Social marketing; Prediction model; Machine learning method; AUC

\section{Highlights}

- The intervention with printed reminder was effective for improving participation of general health check-up.

- In particular, the intervention was most effective among the group with moderate participation probability.

- The targeting using the results of prediction model applying machine-learning method was useful for identifying appropriate intervention targets. 


\section{Correspondence to:}

\section{Akihiro Shimoda, MPH}

Department of Clinical Information Engineering,

Division of Social Medicine,

Graduate School of Medicine, The University of Tokyo, Tokyo, Japan.

E-mail: s04416as@gmail.com
EJBI 2018; 14(4):53-60

Received: July 04, 2018

Accepted: September 07, 2018

Published: September 14, 2018

\section{Introduction}

Non-communicable diseases (NCDs) have been the commonest worldwide threat to health in recent decades [1]. Japan initiated the insurer-based system called the specific health check-up and guidance in 2008, aiming to reduce the burden of NCDs [2]. The program includes a review of risks related to metabolic syndrome and behavioural change support for highrisk participants aged 40-74 years. Several countries provide nationwide health check-up programs to promote lifestyle modification and improve health outcomes in the general public $[3,4,5]$, and there are several previous works reporting the effectiveness of general health check-ups on mortality reduction $[6,7]$.

The participation rate has been increasing gradually after the launch of the general health check-up in 2008 in Japan [8], but approximately half of the subject beneficiaries of the insurers do not participate, despite the considerable effort of providers at promotion. Moreover, the participation rate of dependents (family members) of insured persons is significantly lower than that of insured persons. To overcome this situation, the concept of "data health" was proposed by the Japanese Ministry of Health, Labour and Welfare (MHLW) [9]. "Data health" refers to an effective and efficient promotion of healthcare programs using predictive analytics based on available electronic health records (EHRs). This concept derives from the preceding studies concluding that the application of big data to health care is inevitable [10]. Although the providers of the general health check-up have tried to develop interventions incorporating the idea of "data health", the effects of interventions are not clear due to a lack of sufficient evaluation. The providers do not have enough resources including data analysts, funds for intervention costs, and obvious strategies. Therefore, the interventions in the field have been limited to ineffective ones such as letters, emails, and other communications sent to all subjects without tailoring.

One possible strategy for efficient promotion of the general health check-up based on EHRs would be segmentation of subjects using developed prediction models [11]. If we could predict the subjects who are unlikely to participate in general health checkups, we would select the intervention target efficiently, because we only need to intervene for predicted non-participants. Our previous study showed the effectiveness of prediction models applying the machine learning method to identify individuals who are unlikely to participate in the instruction program for participants of the general health check-up diagnosed as at high-risk of metabolic syndrome [12]. However, there is still scarce evidence about the effectiveness of prediction models to identify the effective population in encouraging participation in the general health check-up based on EHRs.

We aimed to identify the population in which encouraging participation in the general health check-up would be helpful using a prediction model based on a machine-learning method. A secondary analysis of data obtained from the health promotion program using a randomized, controlled design, aimed at improving participation in the general health check-up, was performed. The evaluation of a targeted intervention applying predictive analytics would provide important knowledge for practitioners who need to develop effective and efficient strategies for improving participation in the general health check-up with limited resources.

\section{Methods}

\subsection{Study Design}

The present study was a retrospective secondary analysis of an open, two-arm, randomized controlled trial conducted in the Fukuoka branch of the Japan Health Insurance Association as a health promotion program. The description of the health promotion program using a randomized, controlled design adhered to the Consolidated Standards of Reporting Trials (CONSORT) statement, which deals with the reporting of randomized, controlled trials [13].

\subsection{Setting}

This study used data of a health promotion program conducted in the Fukuoka branch of the Japan Health Insurance Association, which is one of the largest health insurers in Japan, between November 2015 and March 2016. It includes approximately $1,700,000$ insured people, including dependents (family members) of insured persons. In short, the Association provides health services mainly to employees working for small and medium-sized enterprises and their families. As a part of the health services, it provides the general health check-ups for dependents aged 40-74 years through the local network of clinics. The participants were eligible for standard health checks, including measurement of height, weight, BMI, abdominal circumference, systolic 
blood pressure, diastolic blood pressure, fasting blood glucose, triglyceride, high-density lipoprotein (HDL) cholesterol, lowdensity lipoprotein (LDL) cholesterol, glutamic oxaloacetic transaminase, glutamate pyruvate transaminase, gammaglutamyl trans-peptidase, and a dipstick urine test for proteinuria, as well as filling the questionnaire asking medical history and lifestyle (Supplementary Table 1). The contents of health checks are determined by MHLW. Diagnosis of the risk of metabolic syndrome was made according to Japanese criteria [14]. The participants received the test results after several weeks through the mail.

\subsection{Research Subjects}

The Association provided general health check-ups throughout the year. The research subjects were extracted from residents who met the following criteria: dependents (family members) of insured persons in the Fukuoka branch of the Japan Health Insurance Association; residents who did not participate in the general health check-up from April 2015 to the end of September 2015 and who had participated at least once in the past five years (2010-2014); and residents of certain area in Fukuoka prefecture aged 40-74 years. The research subjects were randomly divided into the intervention group and the control group. Since the trial was conducted as a health promotion program, the association wanted to allocate as many research subjects as possible to the intervention group within the budget. Eventually 1,911 and 3,294 subjects were selected for the intervention group and the control group, respectively.

\subsection{Procedure}

In April 2015, all eligible subjects for the general check-up received invitation letters by the association through the mail. Subsequently, subjects were identified according to the eligibility criteria stated above. Then, the research subjects were randomly divided into two groups using a permuted block randomization method by statistician in the Association. Allocation concealment was achieved because the statistician used only insurance identification number when performing randomization. The intervention group received a printed reminder saying "you are due to general health check-up" at the end of November 2015, while the control group received nothing. The research subjects did not know which group whey were assigned to. After four months' follow-up, the participation rates of both groups were assessed for each participation probability group (participation probability was calculated by the prediction model described below). Figure 1 shows the flow diagram of the present study.

\subsection{Data Collection and Outcome Measures}

The primary outcome was the participation rate in the general health check-up between December 2015 and March 2016 (four-month period after the intervention). The participation data were compiled as part of the usual record-keeping process of the designated local clinics. Each clinic sent a written notification to the association when a general health check-up was taken. This information was transferred to a medical history form and used to determine participation in the general health check-up.

\subsection{Prediction Model}

\section{Data and outcome}

Data available or generated during general health checkups were used as prediction variables. Data were extracted from the health information system of the Association. All variables used for the prediction model are shown in Table 1. Participation in the general health check-up from April 2015 to March 2016 was predicted.

\section{Data pre-processing}

There were no missing data because general health check-up data without missing values of dependents (family members) of insured persons are necessary for billing to

Supplementary Table 1: The contents of general health check-up.

\begin{tabular}{|l|l|}
\hline Measurement & $\begin{array}{l}\text { Height, weight, BMI, abdominal circumference, systolic blood pressure, diastolic blood pressure, fasting blood glucose, } \\
\text { triglyceride, high-density lipoprotein (HDL) cholesterol, low-density lipoprotein (LDL) cholesterol, glutamic oxaloacetate } \\
\text { transaminase, glutamate pyruvate transaminase, gamma-glutamyl transpeptidase, proteinuria }\end{array}$ \\
\hline Questionnaire & $\begin{array}{l}\text { Anemia, smoking, weight gain, exercise, physical activity, walking speed, weight change, eating habit, drinking, sleeping, } \\
\text { intention to improve lifestyle, intention to undergo instruction }\end{array}$
\end{tabular}

Table 1: All variables used for the prediction model.

\begin{tabular}{|l|l|}
\hline Variable type & Variable \\
\hline Demographics & Sex, age, height, weight, BMI, abdominal circumference \\
\hline Examination results & $\begin{array}{l}\text { Systolic blood pressure, diastolic blood pressure, fasting blood glucose, triglyceride, high-density lipoprotein (HDL) } \\
\text { cholesterol, low-density lipoprotein (LDL) cholesterol, glutamic oxaloacetate transaminase, glutamate pyruvate } \\
\text { transaminase, gamma-glutamyl transpeptidase, proteinuria }\end{array}$ \\
\hline Questionnaire results & $\begin{array}{l}\text { Anemia, smoking, weight gain, exercise, physical activity, walking speed, weight change, eating habit, drinking, sleeping, } \\
\text { intention to improve lifestyle, intention to undergo instruction }\end{array}$ \\
\hline Past participation & Past participation in general health check-up (2010-2014) \\
\hline
\end{tabular}


the social insurance medical fee fund (each medical institution providing general health check-ups inputs the information so that there are no missing data values). All continuous variables were divided into two to five discrete categories with consideration given to the balance between interpretability and reduction in cluster variance. All variables were then dichotomized as dummy variables.

\section{Model generation}

The prediction model was developed based on random forest (RF) [15] algorithms using machine-learning techniques. RF has become popular due to its efficiency. Moreover, it has been used in a wide variety of research studies thanks to its ability to identify important variables as well as its high accuracy of predictive models $[16,17,18,19]$. The model was trained and tested on a randomly partitioned $80 / 20$ percent split of the data with the caret package in R [20]. RF is an ensemble-based technique using multiple decision trees that can approximate functions with any shape. It is one of the most accurate learning algorithms available and generates an internal unbiased estimate of the generalization error by means of the forest building processes. In order to achieve high accuracy, each tree is developed from a random bootstrapped subset of the training dataset based on bagging and provides a classification as the vote for each tree. This procedure is iterated over all trees in the ensemble, and the average vote of all trees is defined as the RF prediction. Whereas different classifiers overfit the data in a different way, these differences are averaged out through voting because the resulting "forest" of trees

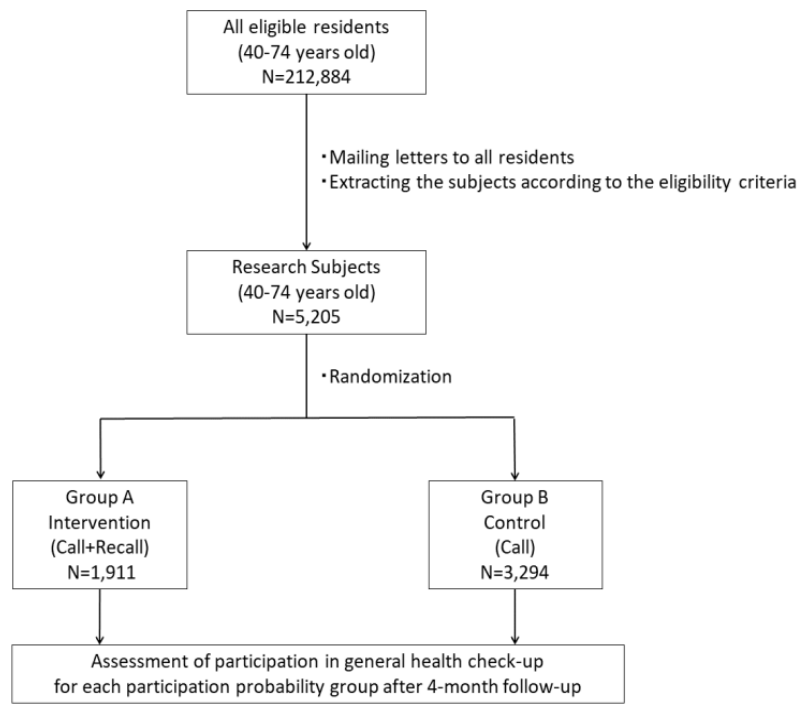

Figure 1: Flow diagram of the trial process.

Table 2: Parameter values of the final model.

\begin{tabular}{|l|l|l|}
\hline Model & Parameter & Value \\
\hline Random forest & num.trees & 500 \\
\hline & mtry & 42 \\
\hline
\end{tabular}

num.trees $=$ Number of trees; mtry $=$ Number of variables used to make one tree. represents the final ensemble model, where each tree votes for the final classification of an individual and the majority wins (i.e., majority voting).

\section{Tuning of Parameters}

The use of RF for predictions required tuning of several parameters. The parameters were determined by a grid search, a method for searching for combinations of parameters. Table 2 shows the tuning parameters and the values of the final model.

\subsection{Statistical Analysis}

To assess the effect of intervention, descriptive analysis was conducted to summarize subjects' age and sex. Chisquared tests were used to determine differences between the intervention group and control group with regards to the participation rate in the general health check-up within four months follow-up period. In addition, to evaluate the effectiveness of intervention targeted by the prediction model, stratified analysis for each of the ten participation probability groups was performed, with intention-to-treat analyses.

Regarding the development of the prediction model, the area under the curve (AUC), the receiver operating characteristic (ROC) curve, sensitivity, and specificity were calculated to evaluate the predictive values of the models using the pROC package in $\mathrm{R}$ [21]. Subsequently, we performed variable importance plot for identifying the most important predictor of future participation in general health check-up with the caret package in $\mathrm{R}$ [20]. All statistical analyses were conducted using $\mathrm{R}$ (Version 3.3.2).

\subsection{Ethical Issues}

The study was approved by the Institutional Review Board of the University of Tokyo (Examination number: 11604) as a retrospective secondary analysis of data obtained from a health promotion program conducted in the Association. The authors used anonymized data.

\subsection{Role of the Funding Source}

There was no funding source for this study. All authors had full access to all of the data. All authors made the decision to submit the manuscript for publication.

\section{Results}

\subsection{Baseline Characteristics of the Research Subjects}

Research subjects in the two groups did not differ in baseline characteristics (Table 3 ). 


\subsection{Performance of the Prediction Model}

The performance of the developed model is shown in Table 4, and the ROC curves of classifiers for the test dataset are shown in Figure 2. The AUC value for test data reflecting the total discriminative ability of the model was 0.668 (95\%CI: 0.635 0.701). Also, sensitivity and specificity of the developed model were 0.538 and 0.720 , respectively.

\subsection{Identification of the Most Important Variable for Prediction}

Figure 3 shows the developed model variable importance plot of the 20 variables exhibiting the highest coefficients of permutation importance for participation in the general health check-up. It revealed that participation in recent years was of high importance.

\subsection{Segmentation of the Research Subjects by}

\section{Prediction Model}

Table 5 shows the distribution of participation probabilities for both groups. Both groups did not differ in terms of participation probabilities.

Table 3: Baseline characteristics of the research subjects by group.

\begin{tabular}{|l|l|l|}
\hline \multirow{2}{*}{ Characteristic } & Group A & Group B \\
\hline & Intervention & Control \\
\hline Sex: & n=1,911 & n=3,294 \\
\hline Male & \multicolumn{2}{|l|}{} \\
\hline Female & $80(4.2)$ & $155(4.7)$ \\
\hline Mean (SD) age (years) & $1,831(95.8)$ & $3,139(95.3)$ \\
\hline Age group (years): & $56.6(9.4)$ & $56.0(9.4)$ \\
\hline $40-49$ & $544(28.5)$ & $1,028(31.2)$ \\
\hline $50-59$ & $547(28.6)$ & $935(28.4)$ \\
\hline $60-69$ & $679(35.5)$ & $1,094(33.2)$ \\
\hline $70-74$ & $141(7.4)$ & $237(7.2)$ \\
\hline
\end{tabular}

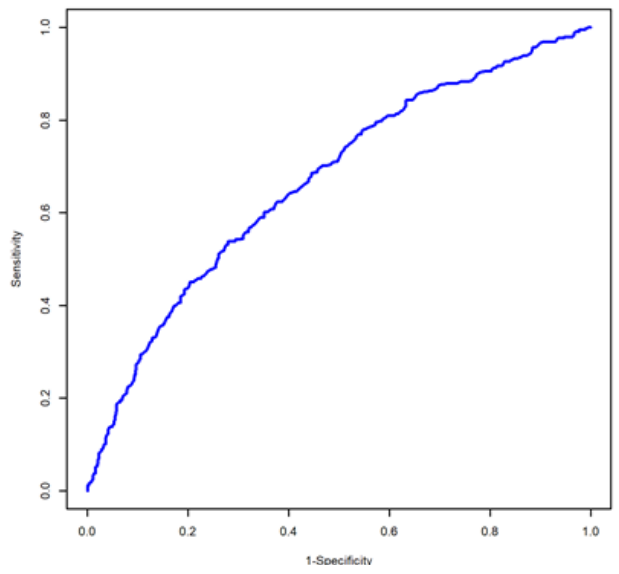

Figure 2: Receiver operating characteristic (ROC) curves for the developed model based on random forest..

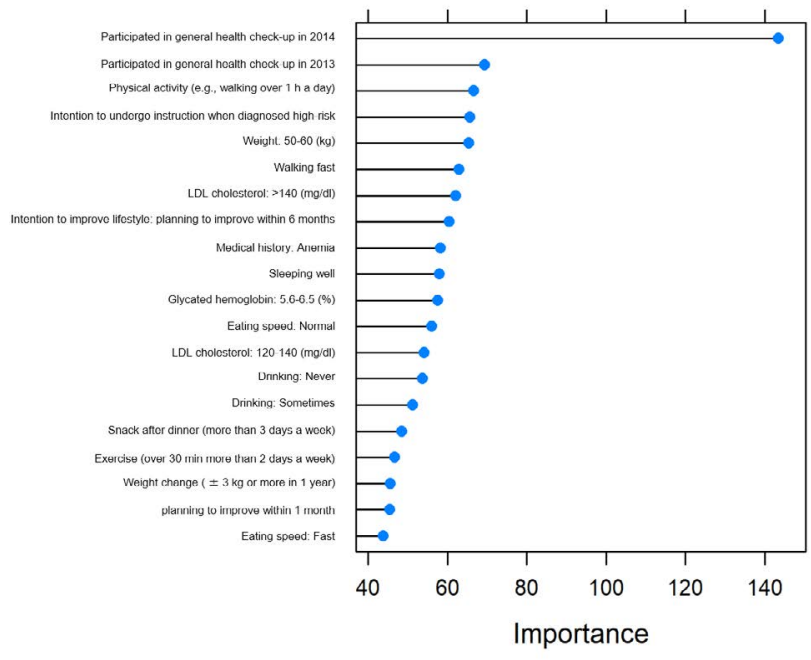

Figure 3: Developed model variable.

Table 4: Performance of the prediction model based on random forest.

\begin{tabular}{|l|l|l|l|l|l|}
\hline Dataset & AUC & $\begin{array}{l}\text { Lower } \\
\mathbf{9 5 \%} \text { CI }\end{array}$ & $\begin{array}{l}\text { Upper } \\
\mathbf{9 5 \% C I}\end{array}$ & Sensitivity & Specificity \\
\hline Training & 1 & 1 & 1 & 1 & 1 \\
\hline Test & 0.668 & 0.635 & 0.701 & 0.538 & 0.72 \\
\hline
\end{tabular}

AUC $=$ area under the curve.

The threshold for calculation of sensitivity and specificity was 0.5 .

Table 5: Distribution of participation probabilities by group.

\begin{tabular}{|l|l|l|}
\hline Participation probability & Group A & Group B \\
\cline { 2 - 3 } & $\begin{array}{l}\text { Intervention } \\
\text { n=1,911 }\end{array}$ & $\begin{array}{l}\text { nontrol } \\
\mathbf{n}=\mathbf{3}, 294\end{array}$ \\
\hline $\begin{array}{l}\text { Mean (SD) participation } \\
\text { probabilities }\end{array}$ & $47.8(25.0)$ & $48.0(24.7)$ \\
\hline Participation probability groups & \\
\hline $0-9 \%$ & $109(5.7)$ & $164(5.0)$ \\
\hline $10-19 \%$ & $247(12.9)$ & $421(12.8)$ \\
\hline $20-29 \%$ & $208(10.9)$ & $342(10.4)$ \\
\hline $30-39 \%$ & $216(11.3)$ & $395(12.0)$ \\
\hline $40-49 \%$ & $204(10.7)$ & $379(11.5)$ \\
\hline $50-59 \%$ & $244(12.8)$ & $412(12.5)$ \\
\hline $60-69 \%$ & $243(12.7)$ & $442(13.4)$ \\
\hline $70-79 \%$ & $230(12.0)$ & $350(10.6)$ \\
\hline $80-89 \%$ & $155(8.1)$ & $285(8.7)$ \\
\hline $90-99 \%$ & $55(2.9)$ & $104(3.2)$ \\
\hline
\end{tabular}

Values are numbers (percentages) unless otherwise stated.

\subsection{The Effectiveness of Intervention for each Participation Probability Group}

Table 6 shows the participation rate in the general health check-up for each participation probability group. Overall, the participation rate of intervention group and control group was $12.4 \%$ and $7.5 \%$, respectively, and there was significant difference between the participation rate of both group $(\mathrm{P}<0.001)$. When stratified by participation probability group, a significant 
Table 6: Participation rate in the general health check-up for each participation probability group.

\begin{tabular}{|c|c|c|c|}
\hline \multirow{3}{*}{$\begin{array}{l}\text { Participation } \\
\text { probability }\end{array}$} & Group A & Group B & \multirow{3}{*}{ P-value } \\
\hline & Intervention & Control & \\
\hline & $n=1,911$ & $\mathrm{n}=3,294$ & \\
\hline \multicolumn{4}{|c|}{ Participation probability groups } \\
\hline $0-9 \%$ & $8 / 109(7.3)$ & $5 / 164(3.0)$ & 0.18 \\
\hline $10-19 \%$ & $15 / 247(6.1)$ & $15 / 421(3.6)$ & 0.187 \\
\hline $20-29 \%$ & $15 / 208(7.2)$ & $22 / 342(6.4)$ & 0.859 \\
\hline $30-39 \%$ & $22 / 216(10.2)$ & $16 / 395(4.1)$ & 0.005 \\
\hline $40-49 \%$ & $25 / 204(12.3)$ & $19 / 379(5.0)$ & 0.003 \\
\hline $50-59 \%$ & $37 / 244(15.2)$ & $32 / 412(7.8)$ & 0.004 \\
\hline $60-69 \%$ & $38 / 243(15.6)$ & $44 / 442(10.0)$ & 0.039 \\
\hline $70-79 \%$ & $34 / 230(14.8)$ & $44 / 350(12.6)$ & 0.523 \\
\hline $80-89 \%$ & $30 / 155(19.4)$ & $34 / 285(11.9)$ & 0.051 \\
\hline $90-99 \%$ & $13 / 55(23.6)$ & $17 / 104(16.3)$ & 0.366 \\
\hline $\begin{array}{l}\text { All research } \\
\text { subjects }\end{array}$ & $237 / 1,911(12.4)$ & $248 / 3,294(7.5)$ & $<0.001$ \\
\hline
\end{tabular}

Each value is the number of subjects who actually participated/the number of subjects in the participation probability group.

difference between the intervention group and control group was seen only for the moderate participation probability groups as follows: $30-39 \%(\mathrm{P}=0.005), 40-49 \%(\mathrm{P}=0.003), 50-59 \%(\mathrm{P}=0.004)$ and $60-69 \%(\mathrm{P}=0.039)$.

\section{Discussion}

The most important finding of the present study is that a significant difference in participation in the general health check-up between the intervention group and control group was observed only among the moderate participation probability group. Since there is scarce evidence about the effective targeting using the results of prediction model based on machine learning method in context of health promotion program, the result of the present study yields an important knowledge for practitioners. The result obtained from the present study is indeed interesting for us as we initially proposed to intervene only in the group with low participation probability. The analysis stratified by the participation probability group showed that the intervention targeted at the group with moderate participation probability might be more cost-effective than that targeted at the group with low participation probability. These results should be interpreted carefully, because the stratified analysis was not pre-determined hypothesis testing, but rather a post hoc subgroup analysis. As a cautionary note in preceding studies [22], we cannot exclude the possibility that the result of the stratified analysis in the present study was obtained accidentally. However, at the same time, the result of the present study is useful in suggesting a possible direction for further verification in future research.

Nevertheless, the present results could be supported by the evidence from preceding studies. Regarding the high participation probability group, they may have participated in general health check-ups more times than the other groups: experience or "enactive attainment", the experience of mastery, is the most influential factor of self-efficacy proposed by Bandura, referring to a personal judgement of how well one can execute action required to deal with prospective situations [23]. Further, Stajkovic et al. found that expectations of self-efficacy determine whether an individual will be able to exhibit coping behaviour and how long effort will be sustained in the face of obstacles [24]. Considering these works, the high participation probability group in the present study maintained a high degree of self-efficacy by past participation in general health check-ups, resulting in returning to participate regardless of exposure to intervention.

On the other hand, regarding the low participation probability group, they possibly have less experience of the general health check-up than other groups. Since the group with low participation probability might represent a population indifferent to health behaviour [25] or those with low socioeconomic status [26] they are a "hard to reach" population when considering bringing about behaviour change [27], resulting in no effectiveness of intervention by postal reminder for participation in the general health checkup in the present study. To enhance the participation rate of this group, preceding review articles suggest that tailored intervention or multiple interventions are needed $[28,29$, 30]. Since the intervention targeting only certain subjects for whom the intervention is more likely to be effective can result in inequality of participation, and even cause health inequality [31], further research is needed to develop and evaluate an effective method to improve participation in the general health check-up among the low participation probability group.

The results of the present study yielded important new knowledge for healthcare practitioners dealing with implementation of the general health check-up. Since providers of general health check-up such as health insurer, local governments and enterprises straggle to enhance the participation rate under financial constraint, knowledge about strategy to identify cost-effective intervention targets would be beneficial for them. Using readily available data in the field, the developed prediction model applying a machine learning method provided useful segmentation of subjects to select more appropriate intervention targets. Since the data types and format of the general health check-up are determined by the Japanese Ministry of Health, Labour and Welfare [32], the method used in the present study could be applicable for other providers of the general health check-up, including medical insurers and local governments. Moreover, the cost of predictive analytics has decreased significantly in recent years because of several open-source software packages, such as TensorFlow or Azure. Consequently, valuable analyses can be done at low cost, even in the absence of experts. If only limited data can be available, it is also possible to develop a prediction model with certain accuracy by reducing the explanatory variables: the variable importance plot showed 
that recent participation in general health check-up was the most useful variable for prediction in the present study.

Furthermore, the present results have policy implications. Although the Japanese healthcare system has achieved high service quality with fairly low health expenditure [33], national health expenditure increases aggressively every year [34]. As proposed in the concept of "data health" by the Ministry of Health, Labour and Welfare (MHLW), ${ }^{9}$ an effective and efficient promotion of healthcare programs using predictive analytics is of great importance in the field of public health. One problem with the concept of "data health" is the lack of a practical idea of effective and efficient implementation of healthcare programs: each insurer has been seeking effective strategies through trial and error. The results of the present study provide these medical insurers or service providers with a possible effective method for implementing cost-effective interventions to improve participation in the general health check-up, and they may drive the dissemination of interventions incorporating the idea of "data health".

\section{Limitation}

There are several limitations of our study. First, this was a retrospective secondary analysis of a health promotion program conducted in the field. Results based on post hoc analysis need prudent consideration. Further research is needed to verify the hypothesis proposed in the present study. Second, the research subjects were dependents (family members) of insured persons. Since dependents account for $27.3 \%$ of the eligible population for the general health check-up in the Fukuoka branch of the Japan Health Insurance Association and have distinctive characteristics, such as a significantly low participation rate, the generalizability of the results is limited. Third, only the short-term effect of intervention was measured. Future research examining the longterm effect of intervention is needed. Fourth, the developed prediction model has much room for improvement in predictive performance. Other variables including medical records or social determinants might improve the predictive accuracy of the model. Fifth, the method is only applicable for past participants in the general health check-up because data are needed to develop the prediction model and compute the participation probability for each individual. Further research is needed to examine the effectiveness of intervention among the "hard-to-reach" population without any data on improvement of participation.

\section{Conclusion}

The intervention with printed reminder was effective for improving participation of general health check-up among the group with moderate participation probability. The targeting using the results of prediction model was useful for identifying appropriate intervention targets.

\section{Author Contribution}

Akihiro Shimoda and Yoshiyuki Saito are guarantors of this work, contributed to the concept and design of the study, statistically analysed data, drafted the manuscript, and provided critical revision of the manuscript for important intellectual content. Chieko Ooe was involved in the acquisition of data, contributed to the concept and design of the study, and provided critical revision of the manuscript for important intellectual content. Daisuke Ichikawa, Ataru Igarashi, Takeo Nakayama, Toki Saito, and Hiroshi Oyama provided critical revision of the manuscript for important intellectual content.

\section{Declaration of Interests}

We declare no competing interests.

\section{Acknowledgements}

No funding to declare

\section{References}

[1] WHO. Estimates for 2000-2015. World Health Organization; 2017. http://www.who.int/healthinfo/ global_burden_disease/estimates/en/index1.html

[2] Ministry of Health L and W. General nationwide health check-up and instruction system called Tokutei-Kenshin in Japan. http://www.mhlw.go.jp/bunya/shakaihosho/ iryouseido01/info02a.html

[3] Liu H, Liu Y, Wang L, Xu D, Lin B, Zhong R, et al. Prevalence of primary biliary cirrhosis in adults referring hospital for annual health check-up in Southern China. BMC Gastroenterol. 2010; 10: 100.

[4] Dubey V, Mathew R, Iglar K, Moineddin R, Glazier R. Improving preventive service delivery at adult complete health check-ups: the Preventive health Evidencebased Recommendation Form (PERFORM) cluster randomized controlled trial. BMC Fam Pract. 2006; 7: 44.

[5] http://www.nhs.uk/conditions/nhs-health-check/pages/ nhs-health-check.aspx

[6] Khan MMH, Goto R, Sonoda T, Sakauchi F, Washio $\mathrm{M}$, Kobayashi $\mathrm{K}$, et al. Impact of health education and screening over all-cause mortality in Japan: evidence from a cohort study during 1984-2002. Prev Med (Baltim). 2004; 38(6): 786-792.

[7] Hozawa A, Kuriyama S, Watanabe I, Kakizaki M, Ohmori-Matsuda K, Sone T, et al. Participation in health check-ups and mortality using propensity score matched cohort analyses. Prev Med (Baltim). 2010; 51(5): 397-402. 
[8] Implementation status of nationwide general health check-up and instruction system in Japan in 2015. http://www.mhlw. go.jp/file/04-Houdouhappyou-12401000-HokenkyokuSoumuka/0000173093.pdf

[9] Ministry of Health L and W. "data health" by medical insurers. http://www.mhlw.go.jp/stf/seisakunitsuite/bunya/ kenkou_iryou/iryouhoken/hokenjigyou/

[10] Murdoch TB, Detsky AS. The Inevitable Application of Big Data to Health Care. JAMA. 2013; 309(13): 1351.

[11] Wu J, Roy J, Stewart WF. Prediction Modeling Using EHR Data. Med Care. 2010; 48: S106-S113.

[12] Shimoda A, Ichikawa D, Oyama H. Prediction models to identify individuals at risk of metabolic syndrome who are unlikely to participate in a health intervention program. Int J Med Inform. 2018; 111: 90-98.

[13] Schulz KF, Altman DG, Moher D, CONSORT Group. CONSORT 2010 statement: updated guidelines for reporting parallel group randomised trials. BMJ. 2010; 340: c332.

[14] Committee for the Diagnosis of Metabolic Syndrome. Definition and Criteria of Diagnosis of Metabolic Syndrome. J Jpn Soc Int Med. 2007; 94: 794-809.

[15] Breiman L. Random Forests. Mach Learn. 2001; 45(1): 5-32.

[16] Zhang H, Bonney G. Use of classification trees for association studies. Genet Epidemiol [Internet]. 2000; 19(4): 323-332.

[17] Khoshgoftaar TM, Golawala M, Hulse J Van. An Empirical Study of Learning from Imbalanced Data Using Random Forest. In: 19th IEEE International Conference on Tools with Artificial Intelligence (ICTAI 2007); 2007 Oct 29-31; Patras, Greece. IEEE; 2007. p. 310-317.

[18] Strobl C, Malley J, Tutz G. An introduction to recursive partitioning: Rationale, application, and characteristics of classification and regression trees, bagging, and random forests. Psychol Methods. 2009; 14(4): 323-348.

[19] Goldstein BA, Hubbard AE, Cutler A, Barcellos LF. An application of Random Forests to a genome-wide association dataset: Methodological considerations \&amp; new findings. BMC Genet [Internet]. 2010; 11(1): 49.

[20] Kuhn M, Wing J, Weston S, Williams A, Keefer C, Engelhardt A, et al. Package "caret." 2017. https://cran.r-project.org/web/ packages/caret/caret.pdf

[21] Robin X, Turck N, Hainard A, Tiberti N, Lisacek F, Sanchez JC, et al. pROC: an open-source package for $\mathrm{R}$ and $\mathrm{S}+$ to analyze and compare ROC curves. BMC Bioinformatics. 2011; 12(1): 77 .
[22] Randomised trial of intravenous streptokinase, oral aspirin, both, or neither among 17,187 cases of suspected acute myocardial infarction: ISIS-2. ISIS-2 (Second International Study of Infarct Survival) Collaborative Group. Lancet. 1988; 2(8607): 349-360.

[23] Bandura A. Self-efficacy: Toward a Unifying Theory of Behavioral Change. Psychol Rev. 1977; 84(2): 191-215.

[24] Stajkovic AD, Luthans F. Self-efficacy and work-related performance: A meta-analysis. Psychol Bull. 1998; 124(2): 240-261.

[25] Norcross JC, Goldfried MR. Handbook of psychotherapy integration. 2nd ed. Canada: Oxford University Press; 2005.p. 548.

[26] Adler NE, Newman K. Socioeconomic Disparities in Health: Pathways And Policies. Health Aff. 2002; 21(2): 60-76.

[27] Bull ER, Dombrowski SU, McCleary N, Johnston M. Are interventions for low-income groups effective in changing healthy eating, physical activity and smoking behaviours? A systematic review and meta-analysis. BMJ Open. 2014; 4(11): e006046.

[28] Moredich CA, Kessler TA. Physical Activity and Nutritional Weight Loss Interventions in Obese, Low-Income Women: An Integrative Review. J Midwifery Womens Health. 2014; 59(4): 380-387.

[29] Gardner MP, Adams A, Jeffreys M. Interventions to Increase the Uptake of Mammography amongst Low Income Women: A Systematic Review and Meta-Analysis. PLoS One. 2013; 8(2): e55574.

[30] Sohl SJ, Moyer A. Tailored interventions to promote mammography screening: A meta-analytic review. Prev Med (Baltim). 2007; 45(4): 252-261.

[31] Gray AM. Inequalities in Health. The Black Report: A Summary and Comment. Int J Heal Serv. 1982; 12(3): 349380 .

[32] The standard data format of "tokutei-kenshin". The Ministry of Health, Labour and Welfare.

[33] Hashimoto H, Ikegami N, Shibuya K, Izumida N, Noguchi $\mathrm{H}$, Yasunaga $\mathrm{H}$, et al. Cost containment and quality of care in Japan: is there a trade-off? Lancet. 2011; 378(9797): 11741182.

[34] Fukawa T. Elderly Population Projection and Their Health Expenditure Prospects in Japan. Mod Econ. 2017; 8(11): 1258-1271. 